\section{LA TECNOLOGÍA COMO MOTOR DE LA INNOVACIÓN EDUCATIVA. ESTRATEGIA Y POLÍTICA INSTITUCIONAL DE LA UNIVERSIDAD DE ALICANTE}

\author{
Faraón Llorens Largo \\ Universidad de Alicante
}

\begin{abstract}
Technological innovation has to be standarized. It is not just a few-freaks-game to kill boredom experimenting with new ideas and tools from technological advances. Universities must acknowledge and empower innovative good practices. They must profit from these practices and set up strategies to lead all of us to better accomplish their educational mission. However, internal policies of each university should be based on justified fundamentals and must be shared by all its community.
\end{abstract}

This paper is divided in three blocks. The first one, strategies to improve teaching + learning through the use of technology, establishes the fundamentals where the University of Alicante lays on. The second one explains the strategy and institutional policies of the University of Alicante during 2005-2008 period, as something already stated and proven. The third block anticipates planning and master lines for 2009-2012 period. Finally, this paper closes with some deep thoughts not to forget lessons learnt and not to make already known mistakes, thus running the right way.

KEY WORDS: Institutional Policies; e-university; e-learning; teaching based on technology; educational innovation; teaching 2.0; learning technologic ecosystem; open educational resources.

\section{Estrategias de mejora de la ENSEÑANZA+APRENDIZAJE POR MEDIO DE LA TECNOLOGÍA}

\subsection{La eUniversidad}

Llevamos casi treinta años desarrollando la UA (Universitat d'Alacant), inspirándonos en la experiencia de siglos de distintas universidades, estableciendo unas reglas de juego y unos procedimientos que nos permitan lograr nuestros objetivos y cumplir con nuestra misión.

Pero el avance tecnológico ha propiciado la aparición de un mundo digital, complementario al mundo físico en el
TECHNOLOGY AS DRIVING FORCE OF THE EDUCATIVE INNOVATION. STRATEGY AND INSTITUTIONAL POLICY AT «UNIVERSIDAD DE ALICANTE»

RESUMEN: La innovación educativa debe de oficializarse. No es sólo cuestión de unos pocos locos que no tienen otra cosa mejor a la que dedicar su tiempo que experimentar con las ideas y herramientas que los avances tecnológicos ponen a nuestro alcance. Las universidades deben reconocer y potenciar esas buenas prácticas innovadoras. Deben alinearlas con su misión y establecer estrategias que nos conduzcan a su cumplimiento. Pero la política interna de cada universidad debe basarse en unos fundamentos que la justifiquen y debe ser compartida por toda la comunidad universitaria. Asi, este articulo se estructura en tres bloques. Un primer bloque, estrategias de mejora de la enseñanza+aprendizaje por medio de la tecnología, que establece esos principios sobre los que se sustenta la política de la Universidad de Alicante. El segundo bloque está dedicado a explicar la estrategia y política institucional de la Universidad de Alicante en el período 2005-2008, como algo ya establecido y pasado. El tercer bloque avanza el planteamiento y líneas estratégicas para el periodo 2009-2012. El artículo se cierra con unas reflexiones que nos permitan escarmentar de la experiencia y no cometer los mismos errores que han permitido a otros aprender y avanzar en la dirección correcta.

PALABRAS CLAVE: Politicas institucionales; eUniversidad;, eLearning; enseñanza basada en la tecnología; innovación educativa; docencia 2.0; ecosistema tecnológico de aprendizaje; recursos educativos abiertos.

que nos movemos, con caracteristicas especificas y especiales, con nuevas reglas y formas de hacer las cosas. Hemos pasado del átomo al bit, de lo local a lo global. El mundo se está aplanando y determinadas caracteristicas de las nuevas tecnologias están acelerando y potenciando ese aplanamiento. Vivimos en un mundo digital, móvil, personal y virtual. Todo lo que pueda digitalizarse se puede fácilmente manipular, transmitir rápidamente, desde cualquier sitio, a través de múltiples dispositivos y lo puedes hacer tú mismo, exactamente como tú lo quieres y en tu dispositivo personal. Disponemos de herramientas que nos conectan con los demás y favorecen la colaboración, facilitan el trabajo en grupos no tan jerarquizados y permiten la creación de redes sociales. Estamos acostumbrados a 
recibir información de varias fuentes, en distintos formatos y soportes y con acceso prácticamente instantáneo.

Se nos ha abierto el eMundo con todos sus diversos submundos. Así hablamos del eGobierno, de la eAdministración, de la eSanidad, de los eBusiness, del eLearning y de los eContenidos. La universidad del siglo XXI debe, manteniendo toda su historia y experiencia acumulada tras siglos de funcionamiento, adaptarse a los tiempos $y$, por tanto, definir y diseñar la eUniversidad, en nuestro caso la eUniversidad de Alicante o eUA.

Los habitantes de estos nuevos mundos tienen nuevas personalidades. Y se habla de la generación del milenio, de la generación red y de la generación Einstein, en referencia a quienes nacieron en la última década del siglo $\mathrm{XX}$, precisamente los que pisan en este momento nuestras aulas como estudiantes. Así, el colectivo universitario más numeroso (es decir los estudiantes) se mueve mejor que nosotros (los profesores) en este mundo. Los niños y los jóvenes de ahora no tienen las mismas costumbres que nosotros cuando éramos niños. Pasan su tiempo de ocio rodeados de productos tecnológicos: ordenadores, Internet, reproductores mp3, teléfonos móviles y videojuegos. Son la primera generación digital por excelencia, han crecido en la sociedad de la información y están acostumbrados a consumir información en cualquier lugar, a cualquier hora, de múltiples fuentes y en cualquier formato. Son tanto consumidores de información como emisores y fuentes de información en la red. Están acostumbrados a adoptar un papel activo, a crear y a diseñar recursos y a interactuar con usuarios de todo el planeta a través de la red.

Son verdaderos nativos digitales. $Y$ nos encontramos con una paradoja. Estos nativos digitales están siendo educados por bárbaros digitales, o en el mejor de los casos por inmigrantes digitales. Es decir, con suerte tendrán profesores que se han adaptado a los tiempos y conocen la tecnología y hacen un uso adecuado de ella. Pero en la mayoría de los casos se encontrarán con profesores que rechazarán, muchas veces por desconocimiento de su potencial, las tecnologías. $Y$ aunque esto ha sido siempre así y los cambios se asumen lentamente, ¿qué hace que ahora nos preocupemos por ello? Hay dos características de estas nuevas tecnologías que hacen especialmente delicada la situación. Por un lado la rápida penetración. Antes una generación tecnológica se desarrollaba a lo largo de varias generaciones humanas, lo que hacia que la tecnología fuese asentándose de manera menos traumática para sus usuarios. Por el contrario, en la actualidad, una generación de personas sufre varios cambios tecnológicos. Por otro lado la amplia penetración, es decir, afecta a todos los ámbitos de la vida.

\subsection{Enseñanza+aprendizaje $(E+A)$}

Hablo de enseñanza+aprendizaje (con signo más) para significar que enseñanza y aprendizaje son dos caras de una misma moneda, indisolubles, que se complementan y se suman. Tradicionalmente se hablaba de enseñar. Ahora se ha inclinado la balanza hacia el lado de aprender. Hay que tener en cuenta que no existe enseñanza si no hay alguien que aprenda y que lo que cree enseñar el profesor, no siempre es igual a lo que aprende el estudiante. En palabras de Giner de los Ríos, "el prurito cuantitativo del profesor /.../ que imagina que todo lo que él dice, lo enseña". Así, un buen sistema educativo debería disminuir al mínimo esta diferencia entre lo enseñado y lo aprendido.

Se habla de que debemos cambiar el punto de vista y situarnos en el lado del estudiante, de medir el esfuerzo que hay que hacer para aprender y aparecen conceptos como enseñanza centrada en el estudiante, que es la base del crédito europeo. Y si situamos al estudiante en el centro del proceso, nos damos cuenta de que aprende de múltiples fuentes, no únicamente del profesor: de los compañeros, de profesores de otras universidades, de los libros, de Internet, de la televisión... Si damos una vuelta más de tuerca, el objetivo debería ser que se aprenda más de lo que se enseña, sobre todo en el nivel universitario.

Y cuando realmente el estudiante se convierte en protagonista del proceso de enseñanza+aprendizaje, incorporamos metodologías activas al proceso docente. Aprendizaje por construcción o significativo, aprendizaje social, aprendizaje colaborativo o cooperativo y aprendizaje basado en proyectos son algunas de las nuevas metodologías que se manejan en los foros de innovación educativa.

Y si además nos ayudamos de la tecnología como medio de comunicación y de distribución del conocimiento, dejando que sea el alumno quien gestione su propio aprendizaje, 
nos encontramos con el eLearning. 0 con el iLearning si nos basamos en la comunicación a través de Internet o con el bLearning si nos aprovechamos de lo bueno de la docencia presencial y lo bueno de la no presencial. Podemos seguir rizando el rizo y hablar del $m$ Learning cuando utilizamos dispositivos móviles, o de gLearning si nos ayudamos de videojuegos educativos ("computer games"). En fin, existen múltiples $\alpha$ Learning, en función del medio en el que hagamos énfasis.

\subsection{Enseñanza basada en la tecnología}

En este artículo vamos a centrarnos en la enseñanza basada en la tecnología y de qué manera puede ayudarnos a mejorar nuestra labor. Una Universidad moderna no puede permanecer al margen de la constante evolución tecnológica, y en especial, esta evolución tiene que verse reflejada muy directamente en todo el proceso de enseñanza+aprendizaje. La incorporación de tecnología frontera al proceso educativo es esencial, máxime cuando se hace imprescindible un cambio metodológico tan importante y necesario como el que se plantea con el reto del Espacio Europeo de Educación Superior (EEES). Al mismo tiempo, como se ha comentado anteriormente, las tecnologías digitales se han convertido en una fuerza de cambio de la sociedad, en general, y de sus organizaciones, en particular. La sinergia de ambos hitos, EEES y tecnologías, nos puede ayudar a un cambio conceptual y metodológico, basado fundamentalmente en un trabajo más activo, tutorizado, de autoaprendizaje, de trabajo en grupo, colaborativo, etc., por parte del alumnado, apoyado en un continuado seguimiento del aprendizaje por parte del profesorado.

Ya no se trata únicamente de estar alfabetizados digitalmente sino de cambiar de perspectiva y relacionar mucho más directamente los aprendizajes que se producen fuera de la escuela con los que se realizan en la misma. Debemos conectar el mundo real en el que viven nuestros estudiantes con las aulas a las que asisten para aprender. Sólo de esa manera las tecnologías se convertirán en un verdadero agente de cambio. La incorporación de nueva tecnología pasa siempre por distintas etapas de madurez. Según Marc Prensky, las cuatro fases de los procesos de incorporación de tecnología son: jugar con la idea, hacer lo viejo a la manera vieja, hacer lo viejo a la manera nueva y hacer cosas nuevas de modos nuevos. No debemos conformarnos con hacer las cosas viejas a la manera nueva, sino de hacer cosas nuevas de modos nuevos. Sólo así la tecnología se convertirá en un agente de cambio que introducirá una verdadera revolución. La utilización de las tecnologías digitales en la educación debe aportar un valor añadido, ya que las tecnologías son un medio, no un fin en sí mismas. Deben permitirnos aumentar la productividad, es decir, aprender más en el mismo tiempo o aprender lo mismo en menos tiempo o aprender de forma más fácil y agradable. Nos deben permitir acceder de forma más flexible al aprendizaje y mejorar la interacción entre los actores del proceso. Aumentar la capacidad de interconexión de los profesores y alumnos. Tanto de los profesores entre sí, como de los profesores con los alumnos y de los alumnos entre ellos. Las tecnologías pueden ayudarnos a cambiar la metodología docente.

Pero a los expertos en el tema les queda como asignatura pendiente el cálculo de la tasa de retorno de la inversión (ROI), es decir, la evaluación, cuantificable y real, de la mejora del proceso de enseñanza+aprendizaje derivada del uso de la tecnología. Es necesario un análisis cuidadoso de su justificación pedagógica, sus ventajas educativas y las implicaciones prácticas de su incorporación. Este aspecto es crucial a la hora de establecer políticas institucionales de fomento del uso de la tecnología en la docencia, ya que estos estudios nos darán los argumentos para justificar las inversiones necesarias.

El uso de las tecnologías en la docencia ofrece nuevas posibilidades, complementarias a la docencia presencial. En especial, se utilizan para mejorar la calidad, para que el alumno participe más activamente en el proceso de aprendizaje y para hacerla llegar a personas que no pueden acceder a la misma de forma presencial. Se han utilizado distintos términos para describir la enseñanza no presencial, algunos de ellos dando énfasis a la herramienta o el medio que se utiliza (docencia no presencial, on-line, a distancia, eLearning, teleformación, iLearning, enseñanza asistida por ordenador...). Entre la docencia cien por cien presencial y la docencia completamente virtual (o no presencial) hay un amplio abanico de enfoques y posibilidades. No debemos limitarnos a una clasificación binaria (presencial y no presencial) sino que debemos explorar y explotar los niveles intermedios. En particular, la modalidad de enseñanza mixta (bLearning) combina la formación presencial tradicional con las tecnologías, recogiendo lo mejor de la enseñanza a distancia y lo mejor de la enseñanza 
presencial. Se trata de integrar, armonizar, complementar y conjugar los medios, recursos, tecnologías, metodologías, actividades, estrategias y técnicas más apropiadas para satisfacer cada necesidad concreta de aprendizaje, tratando de encontrar el mejor equilibrio posible.

\subsection{Docencia 2.0}

En el año 2004, Tim O'Reilly utilizó el término Web 2.0 para calificar a la segunda generación de la web, que se caracterizaba por una nueva forma de interaccionar con el usuario. En este momento la tecnología había evolucionado lo suficiente para ofrecer herramientas que permitieran que un mayor número de gente gestionaran y publicaran en la red, pasando de consumidores a productores de contenido. Más allá de las herramientas, la esencia de la web 2.0 estaría en la democratización y la participación de los usuarios en el desarrollo de la misma. No tardó en generalizarse el uso de la etiqueta 2.0 y adaptarse a distintos ámbitos, entre ellos el educativo. Este calificativo no hace referencia únicamente al hecho de basarse en la tecnología sino además de fomentar una participación activa en el desarrollo de la misma. En estos momentos es posible aprovechar recursos educativos gratuitos y abiertos, a través de iniciativas como OpenCourseWare.

De forma muy simplificada, podemos decir que el mundo antes era analógico, fijo, aislado, genérico, consumidor y cerrado. Mientras que, por contraposición, ahora el mundo 2.0 o eMundo es digital, móvil, conectado, personal, creador y abierto. En estos momentos, la educación comparte las características del mundo anterior y no se ha adaptado a las del mundo 2.0, es decir el mundo real de nuestros estudiantes. Incluso el elearning no explota todas estas características y aunque es digital y móvil, sigue siendo aislado, genérico, consumidor y cerrado. La educación 2.0 deberá tener en cuenta todas estas características en su conjunto.

\section{Estrategia y política institucional de la Universidad de Alicante EN EL PERÍODO 2005-2008}

Espero haber aportado en el primer apartado de este artículo argumentos suficientes para considerar que la re- novación de los planteamientos, las metodologias y los recursos docentes en los niveles universitarios es una necesidad ineludible y estratégica para una universidad del siglo XXI. Así, junto al impulso del nuevo EEES, las tecnologías digitales se han convertido en una fuerza de cambio. Los centros educativos debemos adaptarnos a esta nueva realidad, y ser conscientes de que la tecnologia digital constituye al mismo tiempo que un importante reto, una gran oportunidad. Aunque las nuevas tecnologías no son un fin en sí mismo, sí que se convierten en un valioso medio para alcanzar los objetivos de la Universidad. No se trata simplemente de estar a la última en tecnología, sino en hacer un uso adecuado y provechoso de la misma (cómo y para qué se utiliza).

En el ámbito educativo, en su sentido amplio, el uso de las nuevas tecnologías debe tener como objetivo la mejora de la calidad de la enseñanza y del éxito escolar, aumentando el rendimiento académico de los estudiantes y la productividad de los profesores. Para ello, por un lado se debe impulsar el uso de herramientas de tratamiento de la información y análisis de datos que ayuden a los gestores y directivos de la Universidad en la organización de las enseñanzas y en la toma de decisiones que favorezcan un aumento de la productividad y la eficacia. Por otro lado, se debe intensificar el uso de las tecnologías en la metodología de enseñanza, promoviendo modelos pedagógicos que se basen en ellas y actuando como catalizadoras de innovaciones educativas.

La tecnología puede significar un importante mecanismo para el cambio haciendo posible el aprendizaje distribuido en el tiempo y en el espacio (especialmente importante para la formación continua y para estudiantes a tiempo parcial que combinan estudios y trabajo), aumentar la capacidad de elección por parte del estudiante (permitiendo combinar y complementar las ofertas presenciales con ofertas no presenciales) y en definitiva redefiniendo el proceso de enseñanza+aprendizaje (favoreciendo el papel activo del estudiante y el aprendizaje en grupo). La tecnología puede ser también una herramienta de transformación profunda del papel del profesor en la forma que organiza y participa en la enseñanza y en la investigación. La integración de las TIC en el sistema educativo proporciona un entorno muy apropiado para la utilización de métodos docentes centrados en el trabajo del estudiante, que aboguen por un aprendizaje significativo, activo, personalizado, colabo- 
rativo y autónomo. El empleo de la tecnología digital enriquece el entorno pedagógico y puede servir de catalizador de innovaciones docentes. Estas mejoras y oportunidades conllevan sin embargo dificultades y retos importantes para los estudiantes, profesores y personal de administración y servicios, a los que habrá que saber dar respuesta.

Con todo esto en mente, tras las elecciones rectorales en diciembre de 2004 y con la toma de posesión del nuevo rector y del equipo de dirección a principios de 2005, se crea el Vicerrectorado de Tecnología e Innovación Educativa, con el objetivo de favorecer la integración de las tecnologías digitales en la Universidad, tanto en el ámbito de la gestión, la investigación como la docencia, poniendo especial énfasis en este último aspecto. Convencidos que estos tiempos de cambio aportarán beneficios a la Universidad, se ha realizado una apuesta decidida por la integración de las nuevas tecnologías en la educación, creando las estructuras y soportes adecuados que puedan ayudar a la comunidad universitaria. Por ello, uno de los primeros pasos dados fue la creación del Área ITE (Innovación Tecnológico-Educativa) dentro del Servicio de Informática (http://www.ua.es/ite).

Dado el acelerado ritmo de desarrollo, expansión y transformación de las tecnologías, se precisa de una planificación estratégica detenida, reflexionada, con visión de futuro y ambiciosa. Es importante reflexionar al principio del mandato rectoral sobre qué se quiere hacer en el ciclo completo, ya que de otra manera el día a día nos mantendrá ocupados y no nos permitirá hacerlo. Así, desde el Vicerrectorado de Tecnología e Innovación Educativa planificamos dedicar el año 2005 a la observación de cómo se estaban haciendo las cosas hasta ese momento, el año 2006 al diseño de los nuevos proyectos, 2007 a la ejecución y puesta en marcha de los mismos y finalizar el mandato en el 2008 con la evaluación del impacto de dichos proyectos.

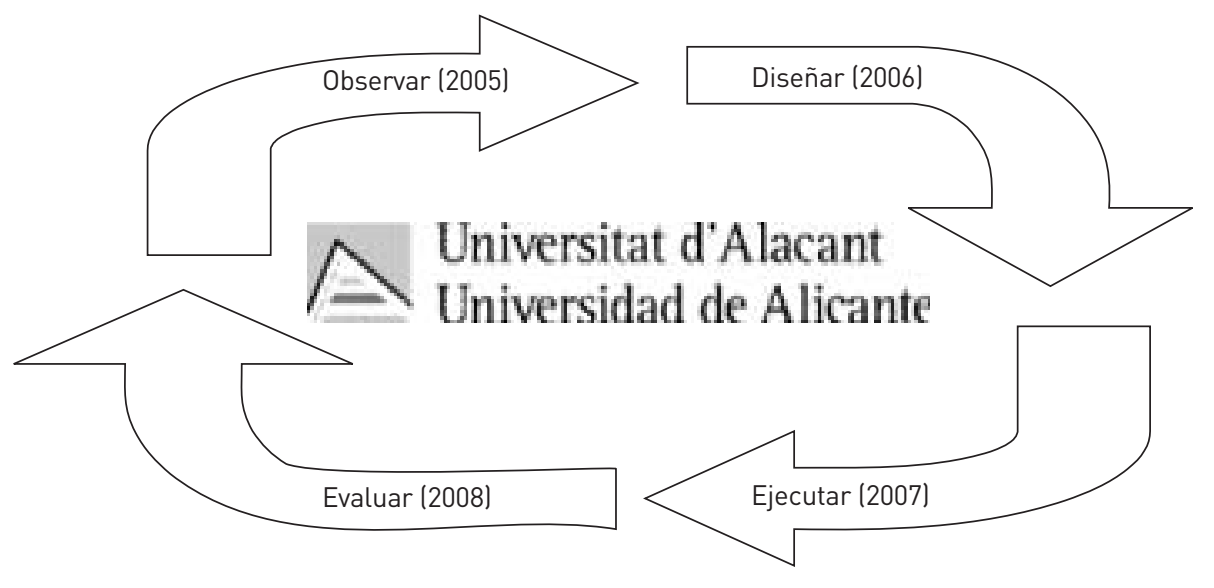

Un aspecto clave de una política institucional de fomento del uso de la tecnología y la innovación educativa es que debe evitar la brecha digital, que podría verse aumentada, entre los profesores que hacen un buen uso de la tecnología y los que no la utilizan, en su mayoría porque la desconocen. Por tanto, las acciones deben ir encaminadas tanto a los convencidos como a los reticentes. A los convencidos que ya hacen un uso avanzado de la misma, a los que la literatura Ilama Ilaneros solitarios, hay que dejarles que vayan por delante y apoyarles institucionalmente en lo que se pueda. A los reticentes hay que ponérselo fácil y mostrarles los beneficios de la misma. En cualquier caso no hay que perder de vista que el objetivo es la mejora del proceso de enseñanza+aprendizaje, en ningún caso el uso por el uso de la tecnología en la docencia. 


\section{1. www.ua.es $\mathbf{2 . 0}$}

En el año 2007, la Universidad de Alicante puso en marcha "www.ua.es 2.0" (http://www.ua.es/es/web20). Bajo esta denominación se agrupa una serie de proyectos y portales. Este nombre se ha elegido por dos motivos. En primer lugar, porque son proyectos que se caracterizan por estar inspirados en la filosofía y principios de la web 2.0, es decir, basados en las comunidades de usuarios, las redes sociales, la interactividad, la participación y colaboración, el contenido multimedia y las herramientas de edición sencillas y fáciles de usar. En segundo lugar, porque estos proyectos han sido definidos y diseñados con posterioridad a la celebración del $10{ }^{\circ}$ aniversario de la web institucional de la Universidad de Alicante (1996-2006), y por tanto, inician su andadura en la segunda década de vida de la misma. Pero estos proyectos no constituyen un fin por sí solos, sino que tienen sentido si se dotan de contenido. Estos portales son ventanas al mundo que ponemos a disposición de la comunidad universitaria para que puedan mostrar sus trabajos y sus opiniones al resto del mundo. El verdadero éxito de estos proyectos lo determinarán las contribuciones de todos los miembros de la Universidad de Alicante.

- Portal AudiovisUAl. Colección de recursos audiovisuales de la Universidad de Alicante (http://cv1.cpd.ua.es/ album).

- RUA. Repositorio Institucional de la Universidad de Alicante (http://rua.ua.es).

- OCW-UA. OpenCourseWare de la Universidad de Alicante (http://ocw.ua.es).

- blogsUA. Weblogs para la comunidad universitaria (http://blogs.ua.es).

- Cicerone UA. Sistema multimedia de navegación por el campus (http://www.cicerone.ua.es).

\subsection{Plan de actuaciones del Vicerrectorado de Tecnología e Innovación Educativa para el año 2008}

(Publicado en el BOUA - Boletín Oficial de la Universidad de Alicante el 21 de febrero de 2008, http://www.boua. ua.es)

El objetivo del Vicerrectorado de Tecnología e Innovación Educativa es conseguir una plena integración de las tecnologías digitales en la Universidad de Alicante, tanto en sus tareas de gestión, de investigación como de docencia. Para ello se debe disponer de infraestructura, equipamientos y aplicaciones informáticas adecuadas. La informatización de la gestión ha sido uno de los primeros aspectos en abordarse y por ello está en un nivel muy alto. La aplicación de las tecnologías digitales a la investigación está también muy avanzada. Su apropiada integración en la docencia ha sido una de las líneas estratégicas para estos años. Es por ello que desde el Vicerrectorado de Tecnología e Innovación Educativa se están Ilevando a cabo acciones para incentivar el uso de las tecnologías digitales en la docencia destinadas a mejorar la calidad del proceso de enseñanza+aprendizaje. Al mismo tiempo, desde este vicerrectorado se está apostando por el conocimiento abierto y fomentando el uso de soluciones de software libre, dadas las ventajas que este reportaría a la Universidad y la creciente calidad del mismo. Con este fin, cumpliendo un ciclo plurianual de actuación de tres años (2006, 2007 y 2008), y tras ser tratadas estas actuaciones en la Comisión de Innovación Educativa, se convocan las siguientes ayudas:

1. Ayudas para la adquisición de software para la docencia instalado en aulas informáticas de uso general.

2. Ayudas directas por la utilización del campus virtual como herramienta de innovación educativa en asignaturas de titulaciones oficiales de primer y segundo ciclo.

3. Convocatoria de proyectos de Innovación TecnológicoEducativa.

4. Ayudas para la creación de Redes Tecnológicas de Innovación Educativa.

5. Ayudas para la creación de Redes Tecnológicas de Introducción de Software Libre en Asignaturas.

6. Ayudas para celebración de encuentros de Innovación Tecnológico-Educativa.

7. Ayudas para la constitución de comunidades de investigación en el Repositorio de la Universidad de Alicante y para incentivar el autoarchivo de documentos por parte de las mismas.

8. Ayudas para la publicación de asignaturas en el OpenCourseWare de la Universidad de Alicante (OCW-UA) y para incentivar el autoarchivo de materiales docentes en repositorio institucional (RUA). 


\subsection{Docencia virtual}

\subsubsection{Docencia virtual en la Universidad de Alicante}

(Presentado al Consejo de Gobierno de la Universidad de Alicante el 26 de septiembre de 2007)

Sin duda, la Universidad de Alicante, siempre a la vanguardia en la incorporación de las tecnologías más innovadora en todas las facetas propias de una institución como la Universidad, necesita en los albores del EEES impulsar y fomentar la innovación tecnológica como medio natural de generar innovación educativa.

Aunque la Universidad de Alicante es una universidad concebida, fundamentalmente, para la enseñanza presencial, es pionera en la aplicación de las tecnologías digitales en su labor (gestión, investigación y docencia), y puede aprovecharse de esta experiencia para mejorar la calidad de su docencia presencial y la captación de estudiantes que tendrian un difícil acceso a una enseñanza totalmente presencial, a través del aprendizaje combinado (bLearning o blendedLearning).

En la enseñanza virtual podemos destacar dos aspectos importantes: la metodología docente y la plataforma tecnológica. Ambos son claves al diseñar un curso virtual, y no debemos caer en el error de que un curso virtual es lo mismo que uno presencial pero puesto en Internet. La enseñanza en entornos virtuales se sustenta en la generación de contenidos, pues es la calidad del material, junto a una metodología participativa y flexible y la labor de tutorización del profesor, lo que determina la valía de un curso de formación on-line. La generación de contenidos para una enseñanza virtual debe considerar tanto las características de la plataforma utilizada como la metodología docente y el estilo de publicación electrónica. La estructura y el diseño de los contenidos juega un papel crucial en este tipo de enseñanzas (organización previa de las ideas generales, organización de los recursos que utilizará el estudiante, programación de contenidos y calendario de actividades,...). En estos momentos la plataforma tecnológica institucional para la docencia virtual en la Universidad de Alicante es el Campus Virtual. Desde el Vicerrectorado de Tecnología e Innovación Educativa se está trabajando en dos líneas: mejora de las herramientas docentes disponibles en campus virtual y análisis de otras plataformas de formación. En este segundo aspecto se dispone de la plataforma Moodle para aquellas asignaturas que, como experiencia piloto, lo soliciten al Vicerrectorado.

Así, concebida más como una metodología en sí misma que como un mero instrumento, la docencia virtual implica un cambio en los roles (nuevos perfiles) de los actores principales del proceso formativo:

- Estudiantes. La docencia en entornos virtuales requiere un modelo de enseñanza activa en el que el estudiante pasa a ser el eje principal del proceso y actor indiscutible de su autoaprendizaje. Pasan de ser consumidores a ser productores de información y conocimiento, lo que requiere una actitud dinámica y una participación activa en todo el proceso.

- Autores. Los autores son los expertos en las determinadas áreas y son los encargados de proporcionar los materiales didácticos, los procesos de evaluación y autoevaluación del alumnado, la guía de estudio y los recursos documentales relacionados (bibliografía, enlaces,...).

- Profesores/tutores. El profesor en un entorno virtual deja de ser un instructor directo para pasar a ser quien facilite y oriente las tareas de aprendizaje. Labores básicas del profesor/tutor son las de orientación, motivación y seguimiento, resolución de dudas (tutorías virtuales) y evaluación continua del estudiante. También, conjuntamente con el autor (responsable/coordinador), definirá el plan docente.

- Equipo Técnico. Personal especializado en la docencia virtual, con conocimientos tanto pedagógicos (metodología docente no presencial) como técnicos (plataforma de formación virtual y formatos digitales). Las principales tareas a desempeñar son formación, apoyo a la generación de materiales multimedia, soporte a Campus Virtual y asistencia técnica. Esta labor será realizada desde el área de Innovación TecnológicoEducativa del Servicio de Informática de la Universidad de Alicante.

\subsubsection{Proyecto piloto para la impartición de asignaturas en modalidad semipresencial}

(Publicado en el BOUA - Boletín Oficial de la Universidad de Alicante el 4 de octubre de 2007, http://www.boua. ua.es) 
Con el objetivo de introducir el uso de las Tecnologías de la Información y la Comunicación (TIC) en la enseñanza reglada y mejorar la calidad del proceso de enseñanza+aprendizaje, el Vicerrectorado de Tecnología e Innovación Educativa presenta este proyecto piloto para la impartición de asignaturas en modalidad semipresencial a través de la plataforma tecnológica que ofrece la Universidad de Alicante.

La aplicación de un modelo semipresencial supone que parte del proceso de enseñanza+aprendizaje se desarrolla en el aula, según el modelo presencial tradicional, mientras que otra parte se basa en la enseñanza on-line, enseñanza virtual o teleformación, permitiendo al alumno disponer de materiales y recursos de apoyo disponibles en línea para una consulta continuada en cualquier momento y de canales para comunicarse con sus compañeros y con el profesorado.

La integración de asignaturas en modalidad semipresencial en la oferta formativa de la Universidad de Alicante ha de estar respaldada por los estándares de calidad. Además, la evaluación de la calidad de la teleformación cobra una especial importancia por su naturaleza. No se debe caer en el error de justificar la teleformación simplemente por estar basada en las TIC. Independientemente de la novedad tecnológica, los contenidos y los procesos formativos asociados a este modelo de enseñanza han de ser cuidadosamente evaluados.

Se propone poner en marcha un proyecto piloto para la impartición de asignaturas en modalidad semipresencial, con las siguientes caracteristicas:

- Incorporación progresiva, a través de proyectos piloto, que posteriormente se irá ampliando a un mayor número de asignaturas.

- Dirigida a asignaturas de titulaciones oficiales de segundo ciclo, asignaturas de másteres oficiales o asignaturas del último curso de una Diplomatura, Ingeniería Técnica o Arquitectura Técnica.

- Tendrán un reconocimiento pleno a todos los efectos (como si se tratase de docencia presencial).

- Dotación económica a la asignatura y reconocimiento en el POI ${ }^{1}$ al profesorado.

- Virtualización parcial de la asignatura. Únicamente se podrá impartir de forma no presencial un número limita- do de créditos (siempre créditos teóricos, que no supere el $50 \%$ de la asignatura y menos de 4,5 créditos).

- Período de implantación: tres cursos académicos.

- Impartida a través de la plataforma tecnológica institucional.

- Con apoyo técnico y pedagógico.

- Con un control y seguimiento de la calidad de la misma.

\subsubsection{Reconocimiento de las tutorías virtuales}

(Publicado en el BOUA - Boletín Oficial de la Universidad de Alicante el 4 de marzo de 2008, http://www.boua.ua.es)

\section{Motivación}

La Universidad de Alicante, siempre a la vanguardia en la incorporación de las tecnologías más innovadora en todas las facetas propias de una institución como la Universidad, necesita en los albores del EEES impulsar y fomentar la innovación tecnológica como medio natural de generar innovación educativa. En esta línea, en el 26 de septiembre de 2007 se presentó en el Consejo de Gobierno el marco de trabajo para la Docencia Virtual en la Universidad de Alicante y la convocatoria del proyecto piloto para la impartición de asignaturas en modalidad semipresencial. Como continuación de ese trabajo se presenta ahora la propuesta de reconocimiento de las tutorias realizadas a través de la plataforma tecnológica facilitada por la Universidad y la posibilidad de reservar parte de las horas de tutorías del profesor para realizarlas virtualmente. El objetivo de introducir el uso de las Tecnologías de la Información y la Comunicación en la enseñanza reglada es mejorar la calidad del proceso de enseñanza+aprendizaje, fomentar la participación activa del estudiante y flexibilizar el acceso de los estudiantes a los recursos que ofrece la Universidad.

La Universidad de Alicante pondrá a disposición de la comunidad universitaria, desde Campus Virtual, las herramientas adecuadas para el control y seguimiento de las tutorias virtuales, tanto por parte de los estudiantes y del profesorado como por parte de los responsables académicos.

\section{Propuesta aprobada}

Hasta un máximo de un tercio de las horas de tutorias se podrán justificar para atención a las tutorias realizadas 
por los estudiantes mediante la herramienta institucional Campus Virtual.

El profesor que opte por justificar parte de su horario de tutorías para atención a las tutorías realizadas a través de Campus Virtual se compromete a:

- Fomentar entre sus estudiantes el uso de la herramienta de tutorias de Campus Virtual.

- Responder, en un tiempo máximo de 48 horas (2 dias laborables), las tutorias que se realicen a través de la herramienta de Campus Virtual.

\section{Planteamiento y líneas estratégicas para el PERíodo 2009-2012}

En el año 2008 hubo de nuevo elecciones rectorales y la comunidad universitaria confió una vez más en el proyecto del equipo rectoral que había estado pilotando la Universidad los últimos cuatro años. Por tanto, en estos momentos acabamos de iniciar una segunda etapa, de forma que apoyándonos en los cimientos creados pretendemos proyectar estos principios estratégicos hacia futuro. El ciclo que se cerraba en el año 2008 ahora debe ser abierto y convertido en espiral. Reconvertimos las tareas del año 2008 y lo dedicamos a replanificar. Dedicaremos 2009 a evaluar los proyectos puestos en marcha y 2010 al rediseño de los mismos, basándonos en la evaluación hecha. Finalmente, 2011 será dedicado a la consolidación de los mismos para darle continuidad más allá de un equipo rectoral. El año 2012 será dedicado a preparar la retirada y deleitarse con la recogida de frutos.

A lo ya construido se van a añadir dos pilares para la sustentación de la política institucional relacionada con la innovación educativa: el ecosistema tecnológico de aprendizaje y los grupos de innovación tecnológico-educativa.

\subsection{Ecosistema tecnológico de aprendizaje}

La fuerza cada vez mayor del movimiento y la existencia de un número creciente de proyectos de recursos educativos abiertos hace que cada vez sea más fácil la creación de ecosistemas abiertos de aprendizaje participativo. $Y$ de espacios públicos educativos. Así, cada uno de nosotros (tanto estudiantes como profesores) dispondrá tanto de un entorno institucional de aprendizaje, facilitado por la universidad, como de un entorno personal de aprendizaje. En el mundo 2.0 es más fácil que grupos de profesores innovadores exploten estas nuevas herramientas, tanto si las instituciones las ponen a su disposición como si utilizan las de dominio público.

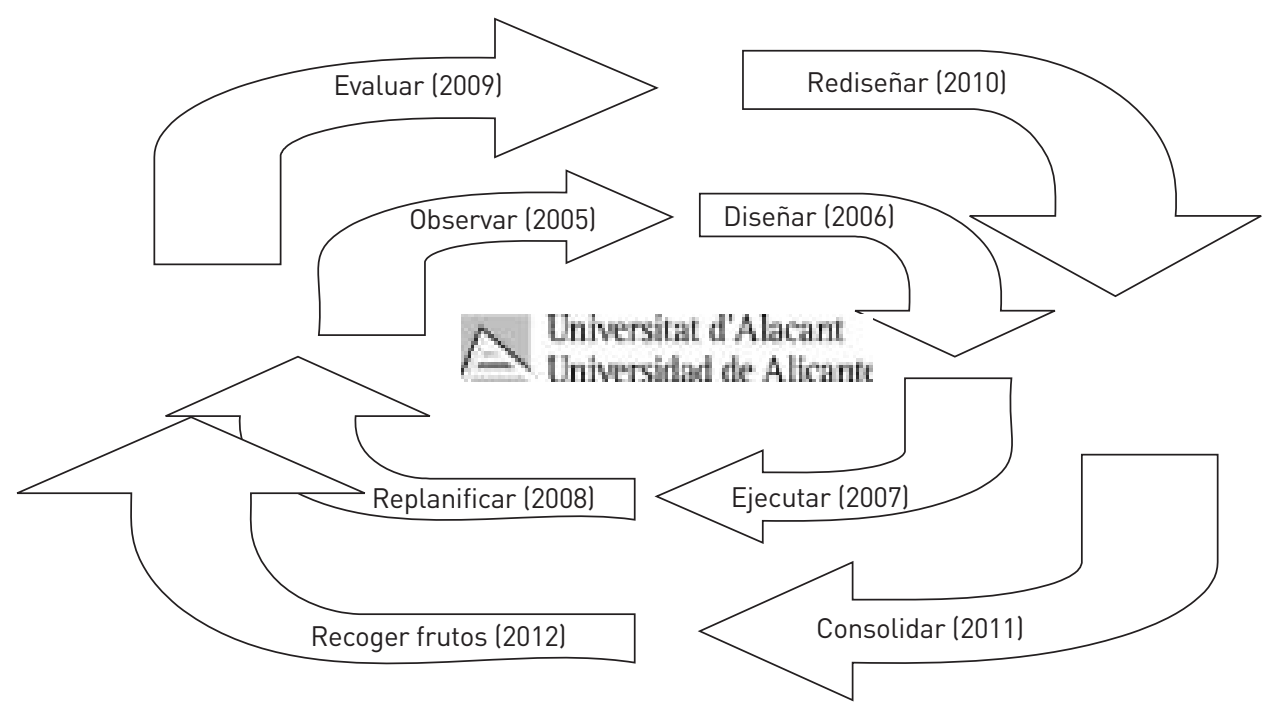


Los campus virtuales y otras herramientas existentes de aprendizaje virtual, siendo bastante completas y útiles en la relación entre profesores y alumnos, por su concepción inicial, son demasiado rígidas y únicamente permiten flujos de comunicación preestablecidos, limitando mucho las posibilidades de interacción. No se trata de prescindir de ellas, sino más bien de complementarlas con otras herramientas, existentes en internet o facilitadas por la institución, formando un ecosistema tecnológico de aprendizaje.

No se trata tanto de centrar el aprendizaje en una herramienta tecnológica como de proporcionar una diversidad que facilite y permita que cada profesor encuentre y cree un entorno de aprendizaje que se adapte a su forma de enseñar.

Una de las características de la anteriormente comentada web 2.0 es precisamente la remezcla ("mashup"), entendida como la reutilización de información de diversas fuentes para crear un nuevo servicio en base a ellas, de forma que tanto el docente como el alumno pueden recoger conocimiento y presentarlo de una manera más adecuada a su situación concreta, permitiendo que se actualice automáticamente en cuanto el servicio original se actualice. Las licencias Creative Commons e iniciativas de conocimiento abierto (Repositorios Institucionales y $\mathrm{OCW}$ ) nos permiten reutilizar y crear nuevos objetos de aprendizaje con un esfuerzo menor.

\subsection{Los grupos de innovación tecnológico-educativa y la acreditación tecnológica}

Las universidades debemos superar la fase del llanero solitario. Si queremos realmente apoyar la innovación educativa deberemos establecer políticas institucionales claras, basadas en principios estratégicos fundamentados y compartidos. La institución debe reconocer el trabajo realizado. La Universidad de Alicante en su afán de alcanzar su objetivo de potenciar la innovación educativa entre su profesorado y sus estudiantes, establece dos ejes estratégicos para este periodo: los grupos de innovación tecnológico-educativa (GITE) y la Acreditación Tecnológica de Profesorado (ATP).

La constitución de un GITE de la UA tiene como objetivo central realizar innovaciones educativas en una o varias líneas de actuación determinadas y seleccionadas por ellos dentro del catálogo establecido. Las líneas de actuación de un GITE no son exclusivas del mismo, pudiendo presentar diferentes grupos las mismas o similares líneas de actuación.
Sin embargo, es objetivo del Vicerrectorado de Tecnología e Innovación Educativa fomentar la creación de grupos que trabajen en líneas similares de forma estable, evitando la atomización de los mismos y procurando la máxima interdisciplinaridad. En este sentido, la agrupación del profesorado de un GITE no está sujeta a ninguna restricción de impartir las mismas asignaturas, adscripción a áreas, departamentos o centros, pudiéndose constituir libremente cumpliendo las condiciones establecidas para ello.

Con el objetivo de dotar estabilidad a los GITE's, el Vicerrectorado de Tecnología e Innovación Educativa mantendrá un registro actualizado de los grupos existentes y las líneas de actuación de cada uno de ellos y la hará pública a través del portal Grupos de Innovación Tecnológico-Educativa de la Universidad de Alicante.

\section{CATÁLOGO DE LÍNEAS DE ACTUACIÓN}

1. Incorporación de tecnología en el proceso enseñanza+aprendizaje

1.1. Uso de herramientas de virtualización (Campus Virtual, Moodle, etc.)

1.2. Tutorias virtuales

1.3. Blogs

1.4. Wiki

1.5. Videoconferencia

1.6. Videostreaming

1.7. OCW-UA (OpenCourseWare de la Universidad de Alicante)

1.8. Otras innovaciones

2. Generación de contenidos didácticos digitales:

2.1. Grabación de videos

2.2. Grabación de audio

2.3. Generación de gráficas animadas

2.4. Generación de presentaciones

2.5. Textos digitales

2.6. Otras innovaciones

3. Generación de recursos digitales interactivos:

3.1. Laboratorios virtuales

3.2. Laboratorios remotos

3.3. Simulaciones interactivas

3.4. Otras innovaciones

4. bLearning

5. Incorporación de software libre en la docencia ( $R$, Scilab, OpenInventor...)

6. Otras innovaciones educativas

(Cualquier innovación educativa no contemplada en los grupos anteriores) 
Entre las condiciones para la constitución de un GITE está la Acreditación Tecnológica de Profesorado (ATP) de la UA. La ATP será computada por primera vez para todos los profesores integrantes de un GITE durante el segundo cuatrimestre del curso 2008/2009 y el primer cuatrimestre del curso 2009/2010. La ATP será realizada con posterioridad siguiendo un calendario similar al establecido inicialmente.

La ATP se obtendrá atendiendo a una valoración hecha en función de los siguientes criterios:

- Empleo de herramientas tecnológicas de innovación educativa. Se obtendrán los puntos directamente del empleo de diferentes funcionalidades asociadas a Campus Virtual: sesiones, materiales, enlaces, dudas frecuentes, tutorias, debates, encuestas, pruebas objetivas, controles y otras funcionalidades docentes. También se tendrá en cuenta el empleo de otras herramientas (Moodle, web propias, etcétera). Pero en este caso, el profesor deberá presentar un informe detallado al finalizar el curso académico en el que se justifique con datos recogidos por el solicitante qué funcionalidades han sido empleadas. Este informe será evaluado por una comisión atendiendo a criterios similares a los empleados para Campus Virtual.

- Uso de tecnología en el aula en consonancia con criterios docentes y pedagógicos, que se valorará mediante informe razonado del profesor. Aspectos a considerar son el uso del ordenador y cañón de proyección disponibles en todas las aulas, la utilización del aula móvil, de proyectores de opacos, de pizarra táctil y de tabletas digitalizadoras, de la funcionalidad del videostreaming y de cualquier otro equipamiento tecnológico.

- Otras experiencias de innovación educativa de carácter avanzado no contempladas en los puntos anteriores. Se valorarán atendiendo a un informe detallado del propio profesor en el que se indique con claridad los objetivos y resultados alcanzados en el terreno de la innovación educativa por medio de la tecnología.

- Asistencia a cursos, talleres o seminarios de innovación educativa.

\section{EscaRMENTAR DE LA EXPERIENCIA}

Para finalizar el artículo y a modo de conclusiones, vamos a dejar constancia de algunas reflexiones. En este sentido "prometo ver la alegría, escarmentar de la experiencia"2. Si, "en cuanto educadores no nos queda más remedio que ser optimistas, jay! Y es que la enseñanza presupone el optimismo tal como la natación exige un medio líquido para ejercitarse" $^{\text {. }}$. Pero al mismo tiempo debemos ser realistas, saber nuestras limitaciones y aprender de la experiencia, tanto de la nuestra como de la de los que nos han precedido. Queremos hacer innovación educativa. Una innovación es un cambio basado en conocimiento, que genera valor. Y dicho cambio resultará más fácil de alcanzar si la Universidad implica a la comunidad universitaria en el proyecto y se dota de los medios tecnológicos y humanos necesarios para poder abordarlos. Elaborar materiales multimedia avanzados, transformar nuestra docencia al concepto bLearning, facilitar los medios y soportes de acceso a la información de los alumnos, etc., será tanto más natural cuanto mayor sea el apoyo dado al profesorado y alumnado en esta línea. Porque "los rectores pueden soñar en grandes visiones de futuro, los vicerrectores, elaborar planes, y los decanos y jefes de departamento, tratar de llevarlos a la práctica, pero sin el apoyo del profesorado nada cambiará. Los profesores sólo cambiarán si pueden ver claramente los beneficios del cambio y las desventajas de no cambiar"4. Para ser aceptadas, las innovaciones deben aportar ventajas, suficientes como para que valga la pena efectuar el cambio. Por eso la innovación es siempre un negocio arriesgado, e incluso una lección de humildad. No hay que cambiar por cambiar, sino mantener lo bueno de lo existente e incorporar aquello nuevo que aporte beneficio. Y "para crear algo nuevo tenemos que ser capaces de mirar la realidad de forma desacostumbrada"5 (creatividad). "La cuestión es cómo equilibrar la profundidad del pensamiento que encontramos en la meditación deliberada de la torre de marfil con la confusión, el ruido y el fugaz entusiasmo del bazar de la educación abierta"6. Debemos dejar paso a las aulas sin paredes y las aulas con paredes transparentes. Cualquier mejora y avance, por pequeño que nos parezca, es bueno. Debemos confiar en su trasmisión y expansión gracias al efecto $m a-$ riposa en la mejora de la docencia universitaria ("el aleteo de las alas de una mariposa se puede sentir al otro lado del mundo"7). El cambio es imparable y no podemos cerrar los ojos al mismo. Buenas políticas institucionales basadas en estrategias claras permitirán a nuestras universidades dar un salto cualitativo. Y finalizamos con el mismo concepto con el que se iniciaba este apartado, la alegría y el optimismo, ya que a fin de cuentas, "la innovación no es más que la inteligencia grupal divirtiéndose"8. 


\section{NOTAS}

Recibido: 26 de febrero de 2009

Aceptado: 16 de abril de 2009
1 Plan de Ordenación Integral de la Universidad de Alicante.

2 Antonio Flores, letra de la canción No dudaría.

5 Juan Mateo, Cuentos que mi jefe nunca me contó.

6 J. Philipp Schmidt, Los recursos educativos abiertos como una estrategia para la apertura y el desarrollo social de la educación superior.

7 Proverbio chino.

3 Fernando Savater, El valor de educar.

4 Tony Bates, Cómo gestionar el cambio tecnológico. Estrategias para los responsables de centros universitarios.
8 Michael Nolan, en Nosotros es más inteligente que yo de Barry Libert y Jon Spector. 